\title{
IS1236, a newly discovered member of the IS3 family, exhibits varied patterns of insertion into the Acinetobacter calcoaceticus chromosome
}

\author{
Ulrike Gerischer, ${ }^{1,2}$ David A. D'Argenio' and L. Nicholas Ornston' \\ Author for correspondence: I. Nicholas Ornston. Tel: +1203432 3498. Fax: +1 2034326161. \\ e-mail: nick-ornston@quickmail.yale.edu
}

1 Department of Biology, Yale University, PO Box 208103, New Haven, CT 06520-8103, USA

2 Institut für

Pflanzenphysiologie und Mikrobiologie, Freie

Universitat Berlin,

Germany

\begin{abstract}
Analysis of spontaneous mutations in Acinetobacter calcoaceticus revealed a 1237 bp insertion sequence named IS1236 and possessing a nucleotide sequence resembling those of members of the IS3 family. The chromosome of A. calcoaceticus strain ADP1 contains seven copies of IS1236 which appears to insert preferentially into pobR, the transcriptional activator of the structural gene for p-hydroxybenzoate hydroxylase. IS 1236 creates tandem 3 bp DNA duplications flanking the sites of its insertion in pobR. Different duplication patterns are found following insertion of IS1236 into pcaH, a structural gene for protocatechuate 3,4-dioxygenase. Therefore the insertion properties of IS1236 appear to be influenced by its DNA target. Amino acid sequences associated with the apparent transposase function have been conserved in ORFB of IS1236 whereas the presumed DNA-binding helix-turn-helix region of IS1236 ORFA exhibits substantial amino acid sequence divergence from its IS3 counterparts. IS1236 ORFA and ORFB coding sequences overlap considerably, and sequence evidence indicates mechanisms for ORFB expression in IS1236 may resemble those employed by other members of the 153 family. Portions of the IS1236 terminal repeats exhibit substantial sequence divergence from other members of the IS3 family, but evolution appears to have conserved a mechanism preventing expression of the insertion sequence genes as a consequence of transcriptional readthrough.
\end{abstract}

Keywords: Acinetobacter calcoaceticus, chromosome, genetic targets, insertion sequence, mutation

\section{INTRODUCTION}

The ease with which Acinetobacter calcoaceticus strain ADP1 undergoes natural transformation makes it a convenient system for analysis of spontaneous mutations. The metabolic pathway used by this bacterium for dissimilation of $p$-hydroxybenzoate is particularly useful for genetic investigation because it is possible to select for strains containing null mutations preventing metabolism of this compound via protocatechuate (DiMarco et al., 1993a, b; Gerischer \& Ornston, 1995). Investigation of such mutations has shown that they all alter DNA within

The GenBank accession number for the sequence reported in this paper is U03772. the $18 \mathrm{kbp} p c a-q u i-p o b$ supraoperonic cluster (Averhoff $e t$ al., 1992; Elsemore \& Ornston, 1994, 1995; Hartnett et al., 1990; Kowalchuk et al., 1994)(Fig. 1).

The most extensive investigation of this genetic system focused upon spontaneous mutations preventing expression of $p c a H$ and $-G$ (Gerischer \& Ornston, 1995). Among 42 sequenced mutations, four were caused by the insertion sequence IS1236 as described in this paper. Other studies had developed procedures for characterizing mutations in either pob $A$ (DiMarco et al., 1993a) or pobR (DiMarco et al., 1993b), so it was possible to assess the relative frequency with which IS1236 is the source of spontaneous null mutations in these genes. As described here, mutations caused by IS1236 in pobR differ from those caused by IS1236 in pcaH in both frequency 

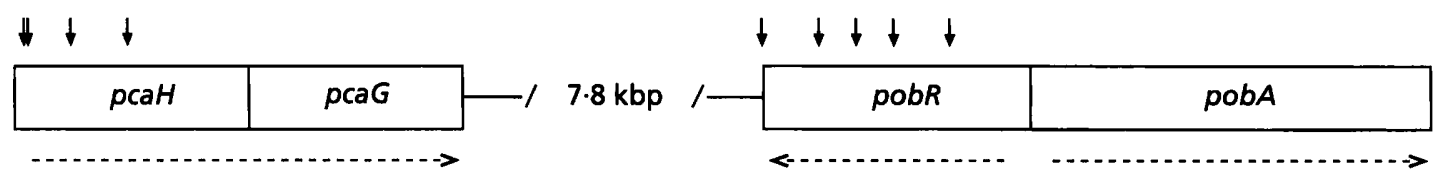

\begin{abstract}
Fig. 1. Chromosomal organization of genes associated with protocatechuate catabolism in A. calcoaceticus. Dashed horizontal arrows indicate directions of transcription. Vertical arrows show positions of IS1236 mutations that were sequenced as part of this study. The respective sizes of pcaH, pcaG, pobR and pobA are 726, 630, 816 and $1215 \mathrm{bp}$. Protocatechuate is converted to citric acid cycle intermediates by enzymes encoded by the pcalJFBDKCHG operon (Kowalchuk et al., 1994). p-Hydroxybenzoate hydroxylase, an enzyme that converts its substrate to protocatechuate, is encoded by pobA (DiMarco et al., 1993a), and the transcriptional activator of this gene is pobR (DiMarco et al., 1993b; DiMarco \& Ornston, 1994). The ends of pcaG and pobR are separated by $7827 \mathrm{bp}$ of DNA largely associated with catabolism of quinate and shikimate to protocatechuate (Elsemore \& Ornston, 1994, 1995; Kloos et al., 1995). Null mutations in pcaB prevent the growth of cells in the presence of protocatechuate or its metabolic precursors (Hartnett et al., 1990). Growth media containing protocatechuate have been used to select from pcaB mutants strains containing secondary mutations blocking expression of $p c a H$ and $-G$ (Gerischer \& Ornston, 1995); similar procedures with $p$ hydroxybenzoate-containing growth media allow selection of strains with secondary mutations blocking expression of either pobA (DiMarco et al., 1993a) or pobR (DiMarco et al., 1993b).
\end{abstract}

and mode of insertion. Analysis of the IS1236 nucleotide sequence reveals biochemical traits conserved as it diverged from other members of the IS 3 family.

\section{METHODS}

Growth of cells, selection of mutant strains, and characterization of mutations. Acinetobacter strain ADP1 used in this investigation is the same as strain BD413 which was initially isolated after enrichment culture of a soil sample with butane 2,3-diol. The organism exhibits extraordinary competence as a recipient in natural transformation (Juni \& Janik, 1969), and the capacity to donate DNA that replaces a recipient $\operatorname{trp} E$ allele through transformation has been taken as evidence that the DNA was derived from a member of the genus Acinetobacter (Juni, 1972). Classification of strains within the genus is complex and not completely resolved. According to some widely accepted classification schemes (Bouvet \& Grimont, 1986; Bouvet $e$ t al., 1990; Ino \& Nichimura, 1989), strain ADP1 (or BD413) should not be designated $A$. calcoaceticus. WiedmannAl-Ahmad et al. (1994) have suggested that these schemes have placed relatively slight emphasis on enviromental isolates and have raised the possibility that satisfactory methods for classification of all Acinetobacter strains are not yet available. Until these issues are fully resolved, we provisionally maintain the species designation $A$. calcoaceticus which has been consistently applied to strain ADP1 (or BD413) since its original isolation.

Previously described procedures were used for growth of cells and selection of mutant strains that failed to express either $p i a H$ and $-G$ (Gerischer \& Ornston, 1995) or pob A (DiMarco et al., 1993a, b). As described elsewhere, natural transformation was used to map mutations within $200 \mathrm{bp}$ of known nucleotide sequence in pcaH and $-G$ (Gerischer \& Ornston, 1995), pob $A$ (DiMarco et al., 1993a) and pobR (DiMarco et al., 1993b). PCR was used to amplify chromosomal DNA for direct sequencing (Gerischer \& Ornston, 1995). Between 50 and 100 bp from each end of IS1236 was determined as part of the sequencing of each mutation.

Sequencing of IS1236. DNA fragments containing portions of IS 1236 from either $p c a H 3$ or pcaH10 (Gerischer \& Ornston, 1995) were amplified by PCR, purified from small components by ultrafiltration using Ultrafree-MC Filter Units low-binding cellulose 30000 NMWL, type PLTK (Millipore), and treated with the Klenow fragment of DNA polymerase to create blunt ends (Sambrook et al., 1989). These fragments were cloned into the $S$ maI site of pUC19. The resulting clones were characterized by restriction analysis and nucleotide sequencing. Together with the results of the direct sequencing of insertion sites, this provided the complete IS1236 insertion sequence. Doublestranded plasmid DNA was sequenced by the dideoxy method using a Sequenase version 2.0 kit (USB) with either standard pUC sequencing primers or custom-designed primers (Gerischer \& Dürre, 1993). Homology searches were performed with the BLAST network service (Altschul et al., 1990).

Copy number of IS1236. Southern blotting (Sambrook et al., 1989) was used to estimate the copy number of IS1236 in $A$. calcoaceticus strain ADP1. The probe was the $1.5 \mathrm{kbp}$ insert from plasmid pZR2018 which contains the first 1143 bp of IS1236 and a 442 bp fragment extending from position 27 to 468 in the nucleotide sequence of $p c a H$. A. calcoaceticus chromosomal DNA was digested with eight different restriction enzymes which do not contain predicted cleavage sites within IS1236. Restriction fragments were separated by agarose gel electrophoresis and blotted onto a nylon membrane. DNA fragments hybridizing with the $p c a H$ portion of the probe were identified on the basis of the known $14 \mathrm{kbp}$ nucleotide sequence of the pcaIJFBDKCHGquiBCXA cluster (Elsemore \& Ornston, 1994, 1995; Kowalchuk et al., 1994). Other hybridizing fragments were attributed to IS1236.

\section{RESULTS}

\section{IS1236 is a member of the IS3 family of insertion sequences}

The nucleotide sequence of IS1236 (Fig. 2) revealed characteristics identifying it as a member of the IS 3 family of insertion sequences. A homology search among DNA segments with known nucleotide sequence showed that IS 1236 most closely resembles IS 3 and IS 1133 found in six Enterobacteriaceae species (Chiou \& Jones, 1993; Lawrence et al., 1992). Greatest nucleotide sequence similarity was found in the ORFB sequences which exhibit identity exceeding $50 \%$ over aligned stretches of more than 500 residues. Properties shared by IS 1236 and IS 3 include (i) terminal inverted repetitions of about $40 \mathrm{bp}$ ending in TGATC/GATCA), (ii) a small ORF (ORFA, occupying positions $73-363$ in IS1236, Fig. 2) overlapped in part by 


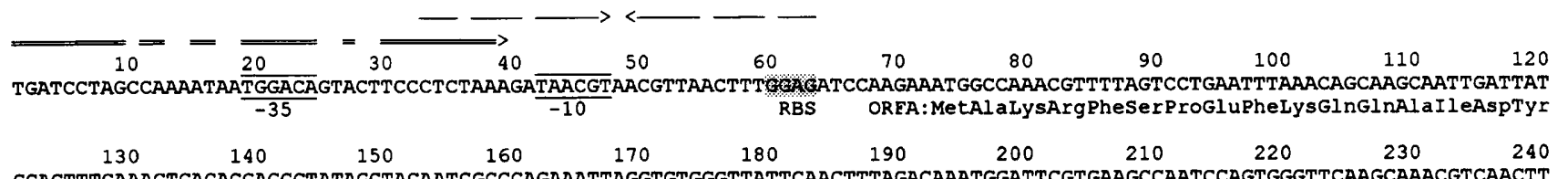
GCACTTTCAAACTCACACGAGCCTATAGCTACAATCGCCCAGAAATTAGGTGTGGGTTATTCAACTTTAGACAAATGGATTCGTGAAGCCAATCCAGTGGGTTCAAGCAAACGTCAACTT

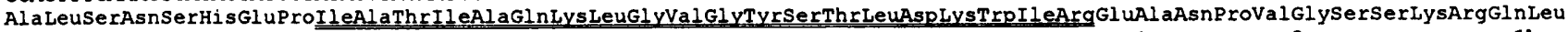

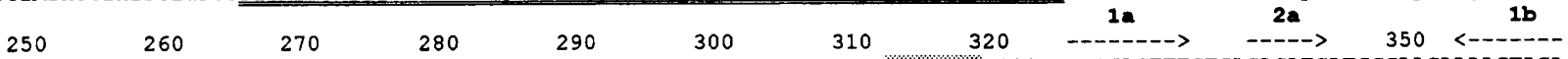
TCACCAGAACAACAGCGGATCTTGGAATTAGAGAAAGAAGTCAAACAGCTCAGGGAAGCCAATGACATCTT) 3 \%

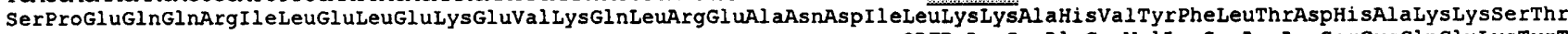

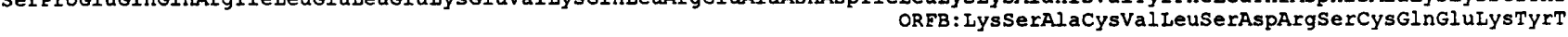

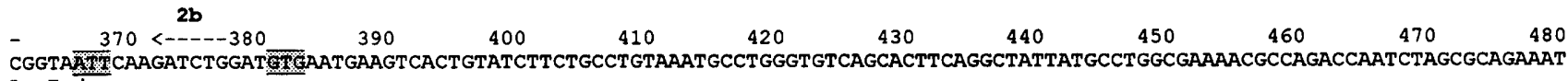
Argend

hrValI leGlnAspLeuAspValAsnGluValThrValSerSerAlaCysLysCysLeuGlyValSerThrSerGlyTyrTyrAlaTrpArgLysArgGlnThrAsnLeUAlaGlnLysT

$\begin{array}{llllllllll}490 & 500 & 510 & 520 & 530 & 540 & 550 & 560 & 570 & 580\end{array}$
ATAATGATTTAAAAGCCGTGATTGGCAGCATCATGCACGATTGGGTGCACCTTCATTGGTACATGACATGCATGATTTAGGTTACAGCATGAGCGAACGACCGTTGGAGAATGCTTA yrAsnAspLeuLySAlaValTyrT rpGlnHi sHi SAl aArgLeuGl yAl aProSerLeuValHi sAspMetHisAspLeuGlyTyrSerMetSerGIuArgThrValGI yArgMetLeuL $\begin{array}{llllllllll}610 & 620 & 630 & 640 & 650 & 660 & 670 & 680 & 690 & 700\end{array}$ AAAAACTTGGTTTACGGAGCAAAATTGCACGTAAATACAAGCATACGACT GATTCAAACCATCGTTTGCCTACAGCGCCTAACTTGTTGGATCGTCAATTTACGGTCAATGAACCCAATA

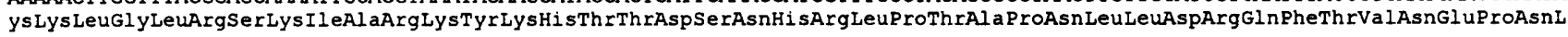
$740 \quad 750$
760
770
780
790
800
810
820
830
840

ATCTGGACAACGGATATTACCTATATCCGTACTAAGCAAGGTTGGCTGTATTTATGTGT GATGCTGGATCTATTCAGCCGTCGTATTGTCGGTTGGCAAACCAGCCATCGAATAGACC ysIleTrpThrThrASPIleThrTyrIleArgThrLysGlnGlyTrpLeuTyrLeuCysValMetLeuAspLeuPheSerArgArgIleValGlyTrpGlnThrSerHisArgI leAspA

$\begin{array}{llllllllll}850 & 860 & 870 & 880 & 890 & 900 & 910 & 920 & 930 & 940\end{array}$ GTCAATTGGTTGTGATGCGTTTCATTATGCAATGGCTCGTCAGGGGTATCCAATGGGTGTCATGGTACATTCGGACCAAGGCTCACAGTACTGTAGTCGTGATTTTAGGGCGCTATTAT

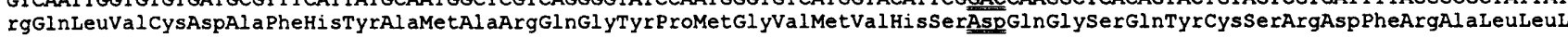
970
980
$990 \quad 1000$
$1010 \quad 1020$
1030
1040
1050
1060
1070
1080

TGACGAATAACTGCGTTCAAAGTATGTCACGCAGGGGAAACTGTTGGGATAATGCAGTGACCGAAAGCTTCTTTCATACATTGAAAGGTCATATGGTCCATGGCAGTGTGTTTGCCACTC euThrAsnAsnCysValGinSerMetSerArgArgGlyAsnCysTrpAspAsnAlaValThrGlú SerPhePheHisThrLeuLysGlyHi sMetValHisGlySerValPheAlaThrA
1090
1100
1110
1120
1130
$1140 \quad 1150$
1160
1170
1180
1190
1200

GAAAAGAAGCGATGCTGTCTTGTTTGACTATATTGAGATTTATTACAATCGAATCAGACGACATTCTACAAACGGCTGGTTAAGCCCAGAAGCCTTTGAACAGAAATATTTCAAGAATT

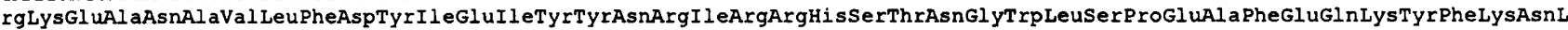
$1210=\frac{1220}{=}=$

TAGAGGGATTTGTTGTCCACGATACTGTCTAGGATCA

euGluGlyPheValValHisAspThrValend

Fig. 2. Nucleotide sequence of IS1236 and the deduced amino acid sequences of ORFA and ORFB. Positions inferred to correspond to positions -35 and -10 with respect to the transcriptional start are overlined and underlined. A likely RBS for ORFA is shaded; there is no obvious RBS for ORFB. Double-lined arrows indicate the inverted repetitions at the termini of IS1236, and single-lined arrows mark an inverted repetition that may block translation of transcripts initiated outside the insertion element (Timmerman \& Tu, 1985). Double-underlining marks a putative helix-turn-helix motif with a highly significant standard deviation score of 5.3 based on the Dodd-Egan weight matrix (Dodd \& Egan, 1990). Acidic amino acid residues that appear to be conserved components of a catalytic triad conserved in IS3 family members and retroviral integrases are double-underlined and overlined (Fayet et al., 1990; Polard \& Chandler, 1995). Dashed arrows accompanied by lettered numerals depict a region of possible RNA secondary structure following a potential ribosomal frameshifting window ('AAAAAAA', shaded and underlined); assignment of these structures is based upon analogy with the corresponding portion of the IS3 nucleotide sequence (Sekine et al., 1994). Potential initiation codons for IS1236 ORFB ('AT' and 'GTG') are shaded, underlined and overlined. It should be noted that nucleotide sequence variations may be exhibited by different copies of IS1236 in the A. calcoaceticus chromosome.

a larger ORF (ORFB, occupying positions 306-1229 in IS1230) which is shifted -1 nucleotide in phase of translation, (iii) an inverted repetition that may block the RBS of ORFA (Timmerman \& Tu, 1985), (iv) a putative helix-turn-helix motif in ORFA (Dodd \& Egan, 1990; Prère et al., 1990), and (v) nucleotide sequences that potentially form a RNA pseudoknot encompassing the end of ORFA (Fig. 2).

\section{Frequency of mutations caused by IS1236 in different genes}

Null mutations blocking metabolism of p-hydroxybenzoate could be caused by dysfunctions in either $p o b \mathrm{R}$ or pob $A$ which occupy about $2.2 \mathrm{kbp}$ of chromosomal
DNA (Fig. 1). Of ten independently occurring null mutations isolated as part of this investigation, six were in the $816 \mathrm{bp} p o b R$, and five of these were IS1236 insertions. None of the four mutations in the $1215 \mathrm{bp} p o b A$ was caused by IS1236 insertion. Analysis of 13 mutants independently derived as part of a separate study (Hartnett et al., 1990) revealed six of seven pobR mutations caused by a $1.2 \mathrm{kbp}$ insertion whereas such an insertion sequence appeared in only one of $\operatorname{six} \operatorname{pob} A$ mutations. Preferential appearance of the $1.2 \mathrm{kbp}$ insertion in pob R mutations (11 of 13) contrasts with its relatively low frequency in $p o b A$ mutations (1 of 10). IS1236 also is a relatively infrequent cause of mutations in the $1.4 \mathrm{kbp} \mathrm{pcaH}$ and $-G$ region. Of 42 sequenced mutations in this region, only four were caused by IS1236, and all of these were in pcaH. 


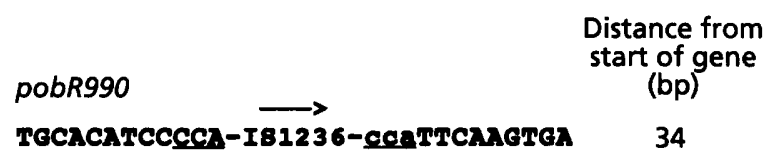

pobR996

AectcoecacAT-I81236-entrracccan

pobR987

AGCAMGATAMTC-I81236-AtaTTCGCGTGA

pobR989

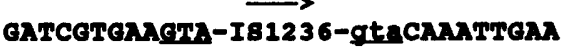

pobR991

ACAGGGGCTrAC-I81236-tacearrecaec

pcaH10

cCNMAGTGeTM-I81236-gttTGCCeATTG

pcaH2

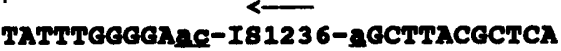

pcaH3

TTGGGGACTTA-I81236-cACGCTCAGCGT

pcaH14

cacorccchrge-I81236-centggcernac
Fig. 3. Target sites and modes of insertion of 151236. The transcriptional orientation of 151236 is indicated by arrows, and a 4 bp deletion in the left terminus of the pcaH14 IS1236 is indicated by the asterisk. Nucleotide sequence newly acquired as a consequence of insertion is shown in lower-case and underlined.

\section{Copy number}

Digestion of the wild-type ADP1 chromosome with ClaI or $\mathrm{XbaI}$ produced seven DNA fragments that hybridized with the IS1236 portion of the probe whereas digestion with AccI produced six such fragments, and four hybridizing chromosomal fragments were produced by EcoRI, HpaII, NarI, SalI or SpbI. The largest hybridizing fragment produced by $S p h I$ was $11 \mathrm{kbp}$. It therefore can be inferred that the chromosome of the $A$. calcoaceticus strain under investigation contains at least seven copies of IS1236. Some of these are separated by no more than $11 \mathrm{kbp}$.

\section{Mode of IS1236 insertion}

The genetic consequences of the IS1236 insertion mutations are summarized in Fig. 3. For the most part, the insertion mutations are fairly evenly distributed throughout $p o b \mathrm{R}$ and $p c a H$. Of the five IS1236 insertion mutations in pobR, three are oriented so that ORFA and ORFB are transcribed in the same direction as the mutated gene, and two carry IS1236 in the opposite orientation. The orientation of the four $p c a H$ inserts is such that ORFA and ORFB transcription is opposed to the transcriptional direction of the mutated gene.

Insertion into pobR invariably produced a $3 \mathrm{bp}$ direct repeat of the target DNA on either side of the insertion sequence, and the terminal inverted repetition of IS1236 was intact in pobR mutations (Fig. 3). Repetitions of $3 \mathrm{bp}$ also flank the insert in $p c a H 10$, but the other three $p c a H$ mutants exhibited different patterns (Fig. 3). In two cases, newly acquired DNA flanking the insertion site could not be traced to a known genetic origin: insertion leading to pcaH2 was accompanied by acquisition of 2 bp corresponding to $\mathrm{AC}$ on the $5^{\prime}$ end of the insert and $1 \mathrm{bp}$ corresponding to $\mathrm{A}$ on the $3^{\prime}$ end of the insert; in $p c a \mathrm{H}^{3}$, 2 bp corresponding to CA lie at the $3^{\prime}$ end of the insert. Most remarkably, the mutation leading to $\mathrm{pcaH} 14$ resulted in a $6 \mathrm{bp}$ direct repetition flanking the insert as well as deletion of the $4 \mathrm{bp}$ from the right terminus of IS 1236 .

Of the nine IS1236 mutations described in Fig. 3, only two (pcaH2 and $p c a H 3)$ failed to give rise to revertants with detectable frequency. Since these two mutations do not include obvious target site duplications, their lack of reversion underscores the importance of flanking direct repeats for IS excision (Galas \& Chandler, 1989). Reversion of pcaH14 suggests that the right terminal TGAT sequence may not be required for excision of IS1236, but precise excision was not determined in this case. Precise excision of IS1236 from pcaH10 was confirmed by direct sequencing of DNA from a revertant strain.

\section{DISCUSSION}

\section{Characteristics shared by IS1236 and other members of the IS3 family of insertion sequences}

Nucleotide sequence comparisons revealed that IS1236 is a member of the IS3 family (Galas \& Chandler, 1989; Timmerman \& $\mathrm{Tu}, 1985)$, with greatest sequence similarity extending over most of ORFB. ORFB amino acid sequences from IS 1236 and Escherichia coli IS 3 share $48 \%$ identity over 221 aligned residues, and conserved within this region is an apparent catalytic triad with invariant aspartate and glutamate residues (Fig. 2). Amino acid sequences containing this triad, believed to be catalytically active, are conserved among transposases of IS3 family members and retroviral integrases (Fayet et al., 1990; Polard \& Chandler, 1995).

Substantial divergence has occurred in the amino acid sequences associated with the helix-turn-helix motif in ORFA. IS 1236 and IS 3 share only 5 of 20 amino acids in this region, but the standard deviation of $5 \cdot 3$ determined with the Dodd-Egan weight matrix for this portion of IS1236 ORFA makes it likely that it conserves the DNAbinding function assigned to the helix-turn-helix motif in 
(a)

Right terminal repeat

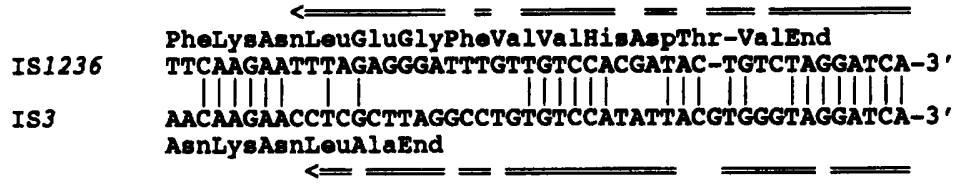

Left terminal repeat

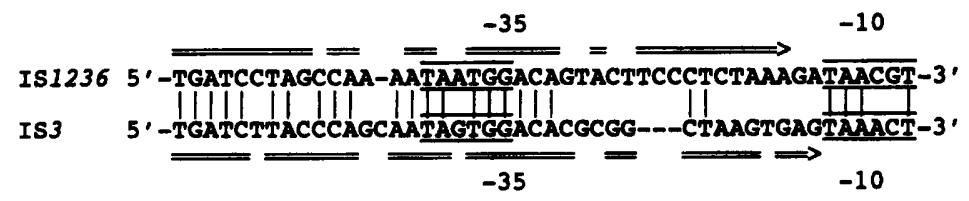

(b)

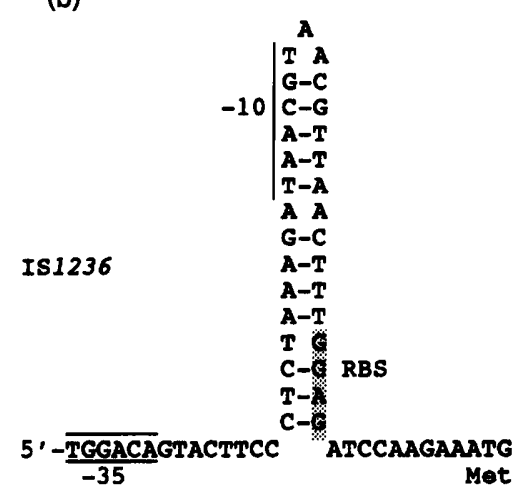

analogous regions of ORFA from other members of the IS3 family.

The nucleotide sequence in the region where ORFA and ORFB overlap (Fig. 2) suggests that the mechanism of IS1236 expression may be similar to that in the three bestcharacterized members of the IS3 family: IS911 (Polard et al., 1991), IS150 (Vögele et al., 1991) and IS3 (Sekine et al., 1994). In these three IS elements, the transposase protein is believed to be an ORFA-ORFB fusion protein produced by a -1 translational frameshift between the overlapping reading frames. As shown in Fig. 2, IS1236 has a potential frameshift window, A AAA AAA, followed by a region of potential RNA secondary structure. The features are in an equivalent position to those shown to be involved in translational regulation in IS3 (Chandler \& Fayet, 1993; Sekine et al., 1994). Furthermore, in IS1236 a potential ORFB initiation codon, AUU, shown to be used in IS911 (Polard et al., 1991), overlaps the ORFA termination codon in the region of potential RNA secondary structure. An analogous situation is found in IS 3 where the RNA secondary structure has been shown to couple translation of ORFB to that of ORFA (Sekine et al., 1994).

The observed copy number of seven for IS1236 in the examined $A$. calcoaceticus strain roughly matches the four to six IS 3 copies observed in laboratory strains of $E$. coli (Galas \& Chandler, 1989), but generalization on the basis
Fig. 4. Divergence of inverted repetitions in is 1236 and IS3. (a) Aligned terminal repetitions. Double-lined arrows indicate potentially hybridizing nucleotides in the coding strand of the respective insertion sequences. Vertical lines mark identical nucleotides in the aligned sequences. Right terminal repeat: coding of IS1236 ORFB extends 24 nucleotides beyond the end of ORFB in the aligned IS3 sequence. Left terminal repeat: horizontal lines mark apparent -35 and -10 bp signal sequences for the start of transcription. Regions containing the -35 bp sequence appear to have been conserved, but DNA downstream from these regions has diverged. (b) Divergence of a hairpin loop that may prevent gene expression from readthrough transcripts. Analogous structures in IS1236 and IS3 may block RBSs (shaded sequences marked RBS) expressed as a consequence of transcriptional readthrough from upstream DNA. If the IS promoters were employed, the transcript would begin with the regions forming the loops which would not be formed (Timmerman \& Tu, 1985). The respective IS1236 and IS3 hairpin loops have diverged so extensively that their $-10 \mathrm{bp}$ regions and RBSs occupy different positions and are protected by different oligonucleotide sequences. Nevertheless, the overall structural requirements for protection against transcriptional readthrough (Timmerman \& Tu, 1985) have been maintained. of this data would be unwise. The $71 \mathrm{E}$. coli natural isolates in the ECOR collection (Pennsylvania State University, USA) averaged significantly less in copies of IS3 (Sawyer et al., 1987). Other investigators, working with different representatives of the same cell line as $A$. calcoaceticus ADP1, found respective IS1236 copy numbers of three and four (M. Stark \& N. Kaplan, personal communication; Zhitomirsky et al., 1994).

In accord with results generally observed with E. coli IS3, sites of IS1236 insertion in pobR are flanked by a $3 \mathrm{bp}$ sequence repetition (Galas \& Chandler, 1989). Only occasionally have variations been observed with $E$. coli: in one study, 2 out of 12 IS 3 insertions resulted in 4 bp target site duplications (Spielmann-Ryser et al., 1991).

\section{Distinctive properties of IS1236}

Behaviour of IS1236 upon insertion is not consistently similar to that generally observed with other IS 3 family members. Three of the four IS1236 insertions in pcaH were not accompanied by acquisition of a flanking $3 \mathrm{bp}$ nucleotide sequence repetition and, in one instance, insertion was accompanied by loss of $4 \mathrm{bp}$ from the left terminus of IS1236 (Fig. 3). Other investigations (Machida \& Machida, 1987) have shown that mutations within an insertion sequence affect the length of duplication introduced at its target site; the present study suggests that the target site itself may influence the length of the duplication. 
The apparent presence of IS1236 in 11 of 13 spontaneous pobR mutations suggests that this gene is particularly susceptible to mutagenesis caused by the insertion sequence. This observation reinforces the conclusion that a general description of IS1236 behaviour will have to take into account properties of its target DNA. Although there is no obvious target site consensus sequence, in eight of the nine observed instances the $20 \mathrm{bp}$ centred on the insertion site is relatively $\mathrm{G}+\mathrm{C}$ rich (45-65 $\mathrm{mol} \%$ ) compared to the mutated gene.

Examination of the respective IS1236 and IS 3 terminal repeats (Fig. 4) reveals substantial divergence. As shown in Fig. 4(a), the amino acid coding function in the IS1236 right terminal repeat extends eight amino acid residues beyond the end of this function in IS3. In the left terminal repeat, extensive nucleotide sequence divergence follows the conserved $-35 \mathrm{bp}$ sequence associated with transcriptional initiation. Fig. 4(b) demonstrates that, although the nucleotide sequences have diverged, they appear to have maintained analogous RNA hairpin structures that may protect ORFA expression against transcriptional readthrough (Timmerman \& Tu, 1985). The trait appears to confer a significant selective benefit because it has been conserved despite extensive nucleotide sequence divergence.

Traits conserved by members of the IS 3 family have been maintained despite substantial differences in the $G+C$ content of divergent insertion sequences. Thus the $\mathrm{G}+\mathrm{C}$ content of IS1236 is $42.2 \mathrm{~mol} \%$, in accord with the respective $G+C$ contents of 42.1 and $42.5 \mathrm{~mol} \%$ for the target genes $p c a H$ and $p o b R$. Such $\mathrm{G}+\mathrm{C}$ contents are typical for $A$. calcoaceticus genes (Shanley et al., 1994; White et al., 1990) and well below those observed for genes from other organisms containing members of the IS 3 family in their chromosomes (Galas \& Chandler, 1989; Lawrence et al., 1992). For example, a member of the IS 3 family in Mycobacterium smegmatis has a $\mathrm{G}+\mathrm{C}$ content of $63.4 \mathrm{~mol} \%$ (Garcia et al., 1994). Such findings indicate that the insertion sequences have diverged from a common ancestor to acquire the $\mathrm{G}+\mathrm{C}$ content characteristic of their respective hosts.

\section{ACKNOWLEDGEMENTS}

U.G. was supported by postdoctoral fellowship $\mathrm{Ge}$ 672/1-1 from the Deutsche Forschungsgemeinschaft (DFG). D.D'A. was a predoctoral fellow supported by the National Institutes of Health. This investigation was supported by grants from the Army Research Office and the General Reinsurance Corporation. We thank M. Stark and N. Kaplan for sharing unpublished information concerning an $A$. calcoaceticus insertion sequence nearly identical to IS1236.

This is publication 13 from the Biological Transformation Center in the Yale Biospherics Institute.

\section{REFERENCES}

Altschul, S. F., Gish, W., Miller, W., Myers, E. W. \& Lipman, D. J. (1990). Basic local alignment search tool. J Mol Biol 215, 403-410.

Averhoff, B., Gregg-Jolly, L., Elsemore, D. \& Ornston, L. N. (1992).
Genetic analysis of supraoperonic clustering in Acinetobacter calcoaceticus. J Bacteriol 174, 200-204.

Bouvet, P. J. M. \& Grimont, P. A. D. (1986). Taxonomy of the genus Acinetobacter with the recognition of Acinetobacter baumanii sp. nov., Acinetobacter bemolyticus sp. nov., Acinetobacter jobnsonii sp. nov., and Acinetobacter junii sp. nov., and emended descriptions of Acinetobacter calcoaceticus and Acinetobacter lwoffii. Int J Syst Bacteriol 36, 228-240.

Bouvet, P. J. M., Jeanjean, S., Vieu, J.-F. \& Dijkshoorn, L. (1990). Species, biotype, and bacteriophage type determinations compared with cell envelope protein profiles for typing Acinetobacter strains. $J$ Clin Microbiol 28, 170-176.

Chandler, M. \& Fayet, O. (1993). Translational frameshifting in the control of transposition in bacteria. Mol Microbiol 7, 497-503.

Chiou, C.-S. \& Jones, A. L. (1993). Nucleotide sequence analysis of a transposon ( $\mathrm{Tn}$ 5393) carrying streptomycin resistance genes in Erwinia amylovora and other gram-negative bacteria. J Bacteriol 175, 732-740.

DiMarco, A. A. \& Ornston, L. N. (1994). Regulation of $p$-hydroxybenzoate hydroxylase synthesis by $p o b R$ bound to an operator in Acinetobacter calcoaceticus. J Bacteriol 176, 4277-4284.

DiMarco, A. A., Averhoff, B., Kim, E. E. \& Ornston, L. N. (1993a). Evolutionary divergence of $p o b A$, the structural gene for $p$ hydroxybenzoate hydroxylase, in an Acinetobacter calacoaceticus strain well-suited for genetic analysis. Gene 125, 25-33.

DiMarco, A. A., Averhoff, B. \& Ornston, L. N. (1993b). Identification of the transcriptional activator $p o b \mathrm{R}$, and characterization of its role in the expression of $p o b A$, the structural gene for $p$ hydroxybenzoate hydroxylase in Acinetobacter calcoaceticus. J Bacteriol 175, 4499-4506.

Dodd, I. B. \& Egan, J. B. (1990). Improved detection of helix-turnhelix DNA-binding motifs in protein sequences. Nucleic Acids Res 18, 5019-5026.

Elsemore, D. \& Ornston, L. N. (1994). The pca-pob supraoperonic cluster of Acinetobacter calcoaceticus contains quiA, the structural gene for quinate/shikimate dehydrogenase. $J$ Bacteriol 176, 7659-7666.

Elsemore, D. \& Ornston, L. N. (1995). Unusual ancestry of dehydratases associated with quinate catabolism in Acinetobacter calcoaceticus. J Bacteriol 177, 5971-5978.

Fayet, O., Ramond, P., Polard, P., Prère, M. F. \& Chandler, M. (1990). Functional similarities between retroviruses and the IS 3 family of insertion sequences? Mol Microbiol 4, 1771-1777.

Galas, J. G. \& Chandler, M. (1989). Bacterial insertion sequences. In Mobile DN A, pp. 109-162. Edited by D. E. Berg \& M. M. Howe. Washington, DC: American Society for Microbiology.

Garcia, M. J., Guilhot, C., Lathigra, R., Menendez, M. C., Domenech, P., Moreno, C., Gicquel, B. \& Martin, C. (1994). Insertion sequence IS1137, a new IS3 family element from Mycobacterium smegmatis. Microbiology 140, 2821-2828.

Gerischer, U. \& Durre, P. (1993). Sequencing using custom designed oligonucleotides. In Metbods in Molecular Biology: DNA Sequencing Laboratory Protocols, pp. 75-81. Edited by H. G. Griffin \& A. M. Griffin. Totowa, NJ: The Human Press.

Gerischer, U. \& Ornston, L. N. (1995). Spontaneous mutations in pcaH,G, structural genes for protocatechuate 3,4-dioxygenase in Acinetobacter calcoaceticus. J Bacteriol 177, 1336-1347.

Hartnett, G. B., Averhoff, B. \& Ornston, L. N. (1990). Selection of Acinetobacter calcoaceticus mutants deficient in pob $A$, a member of a supraoperonic cluster. $J$ Bacteriol 172, 6160-6161. 
Ino, T. \& Nichimura, Y. (1989). Taxonomic studies of Acinetobacter species based on outer membrane protein patterns. $J$ Gen $A p p l$ Microbiol 35, 213-224.

Juni, E. (1972). Interspecies transformation of Acinetobacter: genetic evidence for a ubiquitous genus. J Bacteriol 112, 917-931.

Juni, E. \& Janik, A. (1969). Transformation of Acinetobacter calcoaceticus (Bacterium anitratum). J Bacteriol 98, 281-288.

Kloos, D.-U., DiMarco, A. A., Elsemore, D. A., Timmis, K. \& Ornston, L. N. (1995). Distance between alleles as a determinant of linkage in Acinetobacter calcoaceticus. J Bacteriol 177, 6015-6017.

Kowalchuk, G. A., Hartnett, G. B., Benson, A., Houghton, J. E., Ngai, K. L. \& Ornston, L. N. (1994). Contrasting patterns of evolutionary divergence within the Acinetobacter calcoaceticus pca operon. Gene 146, 23-30.

Lawrence, J. G., Ochman, H. \& Hartl, D. L. (1992). The evolution of insertion sequences within enteric bacteria. Genetics 131, 9-20.

Machida, C. \& Machida, Y. (1987). Base substitutions in transposable element IS1 cause DNA duplication of variable length at the target site for plasmid cointegration. EMBO J 6, 1799-1803.

Polard, P. \& Chandler, M. (1995). Bacterial transposases and retroviral integrases. Mol Microbiol 15, 13-23.

Polard, P., Prère, M. F., Chandler, M. \& Fayet, O. (1991). Programmed translational frameshifting and initiation at an AUU codon in gene expression of bacterial insertion sequence IS 911. J Mol Biol 222, 465-477.

Prère, M. F., Chandler, M. \& Fayet, O. (1990). Transposition in Sbigella dysenteriae: isolation and analysis of IS911, a new member of the IS3 group of insertion sequences. J Bacteriol 172, 4090-4099.

Sambrook, J., Fritsch, E. F. \& Maniatis, T. (1989). Molecular Cloning: a Laboratory Manual, 2nd edn. Cold Spring Harbor, NY: Cold Spring Harbor Laboratory.

Sawyer, S. A., Dykhuizen, D. E., DuBose, R. F., Green, L., Nutangadura-Nhlanga, T., Wolczyk, D. F. \& Hartl, D. L. (1987).
Distribution and abundance of insertion sequences among natural isolates of Escherichi coli. Genetics 115, 51-63.

Sekine, Y., Eisaki, N. \& Ohtsubo, E. (1994). Translational control in production of transposase and in tranposition of insertion sequence IS3. $J$ Mol Biol 235, 1406-1420.

Shanley, M. S., Harrison, A., Parales, R. E., Kowalchuk, G., Mitchell, D. J. \& Ornston, L. N. (1994). Unusual G + C content and codon usage in catIJF, a segment of the ben-cat supra-operonic cluster in the Acinetobacter calcoaceticus chromosome. Gene 138, 59-65.

Spielmann-Ryser, J., Moser, M., Kast, P. \& Weber, H. (1991). Factors determining the frequency of plasmid cointegrate formation mediated by insertion sequence IS 3 from Escherichia coli. Mol Gen Genet 226, 441-448.

Timmerman, K. P. \& Tu, C.-P. D. (1985). Complete sequence of IS3. Nucleic Acids Res 13, 2127-2139.

Vogèle, K., Schwartz, E., Welz, C., Schiltz, E. \& Rak, B. (1991). High-level ribosomal frameshifting directs the synthesis of IS150 gene products. Nucleic Acids Res 19, 4377-4385.

White, P. J., Hunter, I. S. \& Fewson, C. A. (1991). Codon usage in Acinetobacter structural genes. In The Biology of Acinetobacter, pp. 251-258. Edited by K. Towner, E. Bergogne-Bérézin \& C. A. Fewson. New York \& London: Plenum Press.

Wiedmann-Al-Ahmad, M., Tichy, H.-V. \& Schőn, G. (1994). Characterization of Acinetobacter type strains and isolates obtained from wastewater treatment plants by PCR fingerprinting. $A p p l$ Environ Microbiol 60, 4066-4071.

Zhitomirsky, D., Rosenheck, S., Rosenberg, E. \& Kaplan, N. (1994). Isolation and characterization of a novel Acinetobacter insertion element. Acinetobacter'94: Third International Symposium on the Biology of Acinetobacter. Abstract 29.

Received 21 November 1995; revised 29 January 1996; accepted 16 February 1996. 